\title{
Review on flow boiling heat transfer enhancement with nanofluids
}

\author{
Suraj Mali ${ }^{1}$, Ashok Pise ${ }^{1}$, Anil Acharya ${ }^{1}$ \\ ${ }^{I}$ Mechanical Engineering Department, Government College of Engineering, Karad, India
}

\begin{abstract}
There has been increasing interest in nanofluid boiling and its use in heat transfer enhancement. Nanofluids are considered to offer important advantages over conventional heat transfer fluids, researchers focused on measuring the effective thermal conductivity and viscosity of nanofluids.In this paper recent advances in the last decade in flow boiling and convective boiling applications, with nanofluidsalso with studies on CHF increasing by various researchers for convective flow boiling applications using nanofluids as the working fluidare presented. It presents a review of nanofluids, the recent progress on the study of nanofluids, such as the preparation methods, stability of nanofluids, bubble dynamics in flow boiling with the aim of identifying the reasons for its enhancement and the limitations of nanofluid applications based on various published reports.
\end{abstract}

Keywords: nanofluids, critical heat flux, preparation, application.

\section{Introduction}

Nanofluid is a solid-liquid mixture that consists of a nanoparticles and a base liquid[13]. Nanofluids are dilute liquid suspensions of nanoparticles with at least one of their principal dimensions at the Nano scale level [20]. Nanofluids have been found to possess enhanced thermo-physical properties, such as thermal conductivity, thermal diffusivity, viscosity, and convective heat transfer, compared to base fluids, such as oil or water [18]. These particles can be metallic $(\mathrm{Cu}, \mathrm{Au})$, metal oxides $\left(\mathrm{AL}_{2} \mathrm{O}_{3}, \mathrm{~T}_{\mathrm{i}} \mathrm{O}_{2}, \mathrm{~S}_{\mathrm{i}} \mathrm{O}_{2}, \mathrm{Z}_{\mathrm{n}} \mathrm{O}_{2}\right)$, carbon (diamond, nanotubes), or other materials. The typical base fluid alone has a low thermal conductivity. Brownian motion of nanoparticles in the base fluid allows the nanoparticles to maintain their dispersed state and to enhance the thermo-physical properties of the fluid. During nanofluid boiling heat transfer, the nanoparticles deposited on the heating surface can increase the active cavities of nucleate boiling, thereby enhancing boiling heat transfer $[1,7]$.

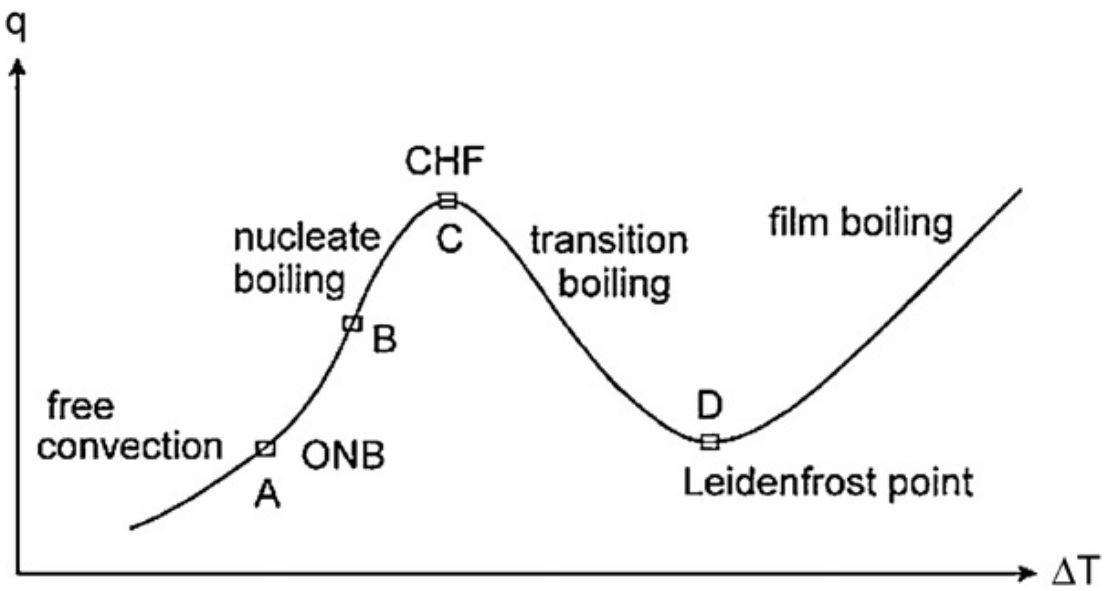

Fig. 1Typical pool boiling curve [3].

Nanofluids are colloidal suspensions of nanoparticles (length scales 1-100 nm) in a base fluid [4, 16, 27]. The critical heat flux (CHF) is defined as the heat flux when the boiling heat transfer coefficient between a heated surface and fluid is dramatically dropped as the phase of fluid near the heated surface changes from liquid to vapour due to a rise of heat flux or surface temperature, or a change of flow rate, pressure, etc. [5,12]. In terms of boiling regimes, nucleate boiling is an efficient heat-transfer mechanism; however, for the incorporation of nucleate boiling in most practical applications, it is imperative that the critical heat flux $(\mathrm{CHF})$ is not exceeded. CHF phenomenon is the thermal limit during a heat-transfer phase change; at the CHF point the heat transfer is maximised, followed by a drastic degradation after the CHF point. Basically, the boiling process changes from efficient nucleate boiling to lesser efficient film boiling at the CHF point. The occurrence of CHF is accompanied by localised overheating at the heated surface, and a decrease in the heat-transfer rate. An 
increase in the CHF of the boiling system would therefore allow for more compact and effective cooling systems for nuclear reactors, air-conditioning units [4].Convective heat transfer can be improved by the addition of nanoparticles to the conventional heat transfer fluids. The concept of nanofluid was proposed by Choiin 1995 . Preparation of nanofluids is an important step in the use of nanoparticles to improve the thermal conductivity of conventional heat transfer fluids.[6]. Flow boiling is affected by additional operational parameters such as flow velocity, flow boiling regimes, quality (and void fraction), surface features, operating pressure, pressure drop in liquid phase and vapour phase[11]. Convective heat transfer can be enhanced passively by changing flow geometry, boundary conditions, or by enhancing thermal conductivity of the fluid. Various techniques have been proposed to enhance the heat transfer performance of fluids. Researchers have also tried to increase the thermal conductivity of base fluids by suspending micro- or larger-sized solid particles in fluids since the thermal conductivity of solid is typically higher than that of liquids [14].The huge number of variables affecting the twophase flow and heat transfer mechanisms: i.e. mass velocity, heat flux, saturation temperature, vapor quality, fluid type, flow direction (vertical, horizontal), gravitational acceleration, vibration, tube diameter, tube length, tube material and surface roughness, presence of additional components in the fluid, nanoparticles, surfactants ,and thermal-hydraulic instabilities.

Compared to conventional solid-liquid suspensions for heat transfer intensifications, properly engineered thermal nanofluids possess the following advantages [2]:

1. High specific surface area and therefore more heat transfer surface between particles and fluids.

2. High dispersion stability with predominant Brownian motion of particles.

3. Reduced pumping power as compared to pure liquid to achieve equivalent heat transfer intensification.

4. Reduced particle clogging as compared to convention slurries, thus promoting system miniaturization.

5. Adjustable properties, including thermal conductivity and surface wettability, by varying particle concentrations to suit different applications.

\section{Nanofluid Formulation.}

The properties and behaviour of nanofluids much depend on the properties of the base liquid and the dispersed phases, particle concentration, size and morphology, as well as the presence of dispersants or surfactants. Formulating stable nanofluids, with controlled properties such as thermal conductivity, viscosity and wettability for heat transfer applications, still presents a challenge for the nanofluid community. Two methods can be generally used for such formulation, namely, the top-down method through size reduction (the two-step method), and the bottom-up approach through simultaneous production and dispersion of nanoparticles (the onestep method) $[2,14]$.

\subsection{The two-step method}

For the two-step method, dried nanoparticles have to be either synthesized or purchased in the form of dry powders. Nanoparticles synthesized through different routes have different morphologies, structures and hence different physical and chemical properties, which will affect the quality of the final nanofluids. The mechanical route, such as milling and grinding, is straight forward but energy intensive. The liquid route such as the sol-gel and wet chemical methods typically involves chemical reactions of a few reagents with one desired product while normally producing other impurities that are difficult to control. The vapour -phase route involves producing a reactive vapor in which nucleation and growth occur through combustion, pyrolys is, plasmas, laser ablation, laser pyrolysis, or chemical vapour deposition. From both economic and quality considerations, the gas phase route has become the most favourable method for large scale production of nanoparticles. Compared to the bottom-up approach, dispersing dry particles into liquids has been more frequently used by researchers in the nanofluid field. The general equipment used in dispersing dried nanoparticles includes ultrasonic baths, magnetic stirrers, high-shear mixers, homogenizers and bead mills. To overcome the stability problem, stabilizers are often introduced during the formulation process, as illustrated schematically in Fig. 3 that involves three steps: (a) preparing pure liquid, (b) mixing suitable stabilizers to the pure liquid (base liquid) and (c) dispersing nanoparticles into the base liquid. In the nanofluid community, the heat transfer capability of nanofluids is generally compared with that of pure liquid, which is quite misleading. The stabilizers, especially those loose ones as shown in Fig. $3 \mathrm{c}$ could alone alter the values of surface tension, wettability and viscosity [2, $14,17,21]$. 


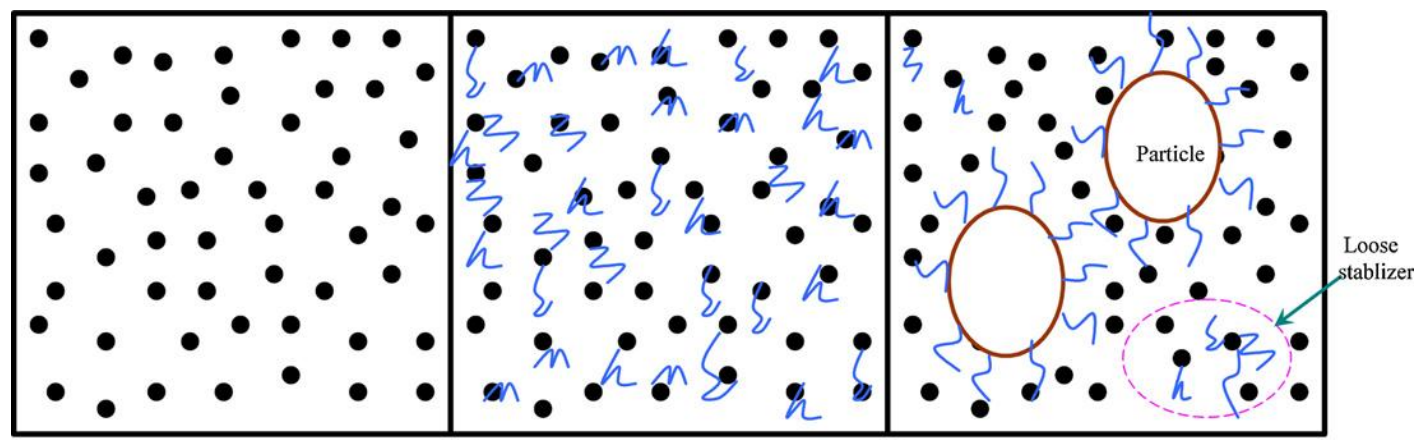

(a) pure liquid

(b) pure liquid + stablizers

(c) nanofluids with loose stablizers in the liquid

Fig. 2 Two-step method for nanofluids formulation [2].

\subsection{The one-step method}

The one-step process consists of simultaneously making and dispersing the particles in the fluid. In this method, the processes of drying, storage, transportation, and dispersion of nanoparticles are avoided, so the agglomeration of nanoparticles is minimized, and the stability of fluids is increased. The one-step processes can prepare uniformly dispersed nanoparticles, and the particles can be stably suspended in the base fluid.One-step physical method cannot synthesize nanofluids in large scale, and the cost is also high, so the one-step chemical method is developing rapidly. However, there are some disadvantages for one-step method. The most important one is that the residual reactants are left in the nanofluids due to incomplete reaction or stabilization. It is difficult to elucidate the nanoparticle effect without eliminating this impurity effect [21].

\section{Stability Of Nanofluids}

The agglomeration of nanoparticles results in not only the settlement and clogging of microchannels but also the decreasing of thermal conductivity of nanofluids. So, the investigation on stability is also issue that influences the properties of nanofluids for application, and it is necessary to study and analyze in fluencing factors to the dispersion

Stability of nanofluids

\subsection{The Stability Evaluation Methods forNanofluids}

Many methods have been developed to evaluate the stability of nanofluids. Those are Sedimentation method, Centrifugation Method, Zeta Potential Analysis, Spectral Absorbency Analysis. The simplest method is sedimentation method. The sediment weight or the sediment volume of nanoparticles in a nanofluid under an external force field is an indication of the stability of the characterized nanofluid. The variation of concentration or particle size of supernatant particle with sediment time can be obtained by special apparatus. The nanofluids are considered to be stable when the concentration or particle size of supernatant particles keeps constant. Sedimentation photograph of nanofluids in test tubes taken by a camera is also a usual method for observing the stability of nanofluids.For the sedimentation

Method, long period for observation is the defect. Therefore, centrifugation method is developed to evaluate the stability of nanofluids. Zeta potential is electric potential in the interfacial double layer at the location of the slipping plane versus a point in the bulk fluid away from addition of a surfactant could improve the stability of the suspensions. Spectral absorbency analysis is another efficient way to evaluate the stability of nanofluids. In general, there is a linear relationship between the absorbency intensity and the concentration of nanoparticles in fluid [21].

\subsection{The Ways to Enhance the Stability of Nanofluids}

Surfactants used in nanofluids are also called dispersants. Adding dispersants in the two-phase systems is an easy and economic method to enhance the stability of nanofluids. Dispersants can markedly affect the surface characteristics of a system in small quantity. Dispersants consists of a hydrophobic tail portion, usually a long-chain hydrocarbon, and a hydrophilic polar head group. Dispersants are employed to increase the contact of two materials, sometimes known as wettability. In a two-phase system, a dispersant tends to locate at the interface of the two phases, where it introduces a degree of continuity between the nanoparticles and fluids. According to the composition of the head, surfactants are divided into four classes: non-ionic surfactants without charge groups in its head (include polyethylene oxide, alcohols, and other polar groups), anionic surfactants with negatively charged head groups (anionic head groups include long-chain fatty acids, sulfo succinates, alkyl sulfates, phosphates, and sulfonates),cationic surfactants with positively charged head groups (cationic surfactants may be protonated long-chain amines and long-chain quaternary ammonium compounds), and amphoteric surfactants with zwitter ionic head groups(charge depends on ph[21]. 


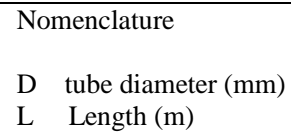

\section{Boiling Heat Transfer Basis}

Boiling is a phase change (from liquid to vapor) process in which vapor bubbles are formed either on a heated surface or in a superheated liquid layer adjacent to the heated surface. Although boiling is a complex and elusive process, it is very efficient mode of heat transfer in various energy conversion and heat exchange systems as well as cooling of high energy density electronic components. There are two common types of boiling: pool boiling and flow or forced convective boiling. Pool boiling refers to boiling on a heated surface(heater) submerged in a pool of initially quiescent liquid while flow boiling is boiling in a flowing stream of fluid, where the heating surface may be the channel wall confining the flow. Heat flux in any boiling is one of the most important parameters in designing and operating the heat transfer equipment's with high heat flux such as boiler, evaporator, electronic equipment and rocket engines [3].

\subsection{FlowBoiling heat transfer of nanofluids-literature survey}

The boiling critical or burnout heat flux enhancement of particle-suspensions depends on the particle concentration, $\mathrm{pH}$ of the solution as well as on the deposition of the particles on the suspended heater surface. It is long back proven that addition of solid particle in base fluid can alter its boiling heat transfer performance. Among studies on flow boiling T. Kim et al.[5] reported about flow boiling CHF enhancement experiments using $\mathrm{AL}_{2} \mathrm{O}_{3}$ /water nanofluids in SS-316 circular tube. The CHFs of $\mathrm{AL}_{2} \mathrm{O}_{3}$ nano-fluids were enhanced up to $70 \%$ in flow boiling. S. Suresh et al. [6] found experimentally for $\mathrm{AL}_{2} \mathrm{O}_{3}-\mathrm{Cu} /$ water hybrid nanofluids in uniformly heated circular tube the convective heat transfer experimental results showed a maximum enhancement of $13.56 \%$.Also S. Z. Heris et al.[9] found the enhancement of heat transfer for $\mathrm{AL}_{2} \mathrm{O}_{3}$ /water nanofluid in circular tube. M.M. Heyhat et al.[10] do experimental investigation of laminar convective heat transfer of water-based $\mathrm{AL}_{2} \mathrm{O}_{3}$ nanofluids in fully developed flow regime found that the heat transfer coefficient ofnanofluid is higher than that of the base fluid and increased with increasing the Reynolds number and particle concentrations. The heat transfer coefficient increases by approximately $32 \%$ in the fully developed region at 2 vol.\% nanofluids. A.A.R. Darzi et al. [24] didexperimental investigation of turbulent heat transfer and flow characteristics ofSiO2/water nanofluid within helically corrugated tubes results show that adding the nanoparticles in tube with high height and small pitch of corrugations augments the heat transfer significantly. R. B. Mansour et al.[7] did experimental study of mixed convection with water $\mathrm{AL}_{2} \mathrm{O}_{3}$ nanofluid in inclined tube results show that the experimental heat transfer coefficient decreases slightly with an increase of particle volume concentration from 0 to 4\%.K.Henderson et al.[12] did experimentation for Flow-boiling heat transfer of R134a-based nanofluids in a horizontal tube results shows With direct dispersion of $\mathrm{S}_{\mathrm{i}} \mathrm{O}_{2}$ nanoparticles in R-134a, the heat transfer coefficient decreases (as much as 55\%) in comparison to pure R-134a, However, excellent dispersion is achieved for a mixture of $\mathrm{R}-134 \mathrm{a}$ and polyester oil with $\mathrm{CuO}$ nanoparticles, and the heat transfer coefficient increases more than 100\%.I.M. Mahbubul et al.[13] studied experimentally heat transfer characteristics of $\mathrm{AL}_{2} \mathrm{O}_{3}-\mathrm{R} 141 \mathrm{~b}$ nano refrigerant in horizontal smooth circular tube found that heat transfer characteristics increased with the enhancement of nanoparticle volume concentrations.

Table 1Summary of experiments on convective heat transfer of nanofluid

\begin{tabular}{|c|c|c|c|}
\hline Investigator & Geometry & Nanofluid & Effect \\
\hline T.Kim et al. [5] & $\begin{array}{l}\text { SS-316, } \mathrm{D}_{0}=12.78[\mathrm{~mm}] \\
\text { Thickness }=0.9[\mathrm{~mm}], \quad \mathrm{L}=0.5[\mathrm{~m}]\end{array}$ & $\mathrm{AL}_{2} \mathrm{O}_{3} /$ Water & $\begin{array}{c}\mathrm{CHF} \\
\text { enhancement } 70 \% \\
\end{array}$ \\
\hline S. Suresh et al. [6] & $\begin{array}{l}\text { Uniformly heatedCircular tubeDim.- } \\
\text { Unspecified }\end{array}$ & $\mathrm{AL}_{2} \mathrm{O}_{3}-\mathrm{Cu}$ & $\begin{array}{c}\mathrm{CHF} \\
\text { enhancement } 13 \% \\
\end{array}$ \\
\hline S. Z. Heris et al.[9] & $\begin{array}{l}\text { Copper tube, } \quad \mathrm{Di}=6[\mathrm{~mm}] \\
\mathrm{L}=1[\mathrm{~m}], \text { Thickness }=0.5[\mathrm{~mm}]\end{array}$ & $\mathrm{AL}_{2} \mathrm{O}_{3} /$ Water & $\begin{array}{c}\mathrm{CHF} \\
\text { enhancement }\end{array}$ \\
\hline M.M. Heyhat et al.[10] & $\begin{array}{l}\text { Copper tube, } \quad \mathrm{D}_{\mathrm{i}}=5[\mathrm{~mm}] \\
\text { Thickness }=0.5[\mathrm{~mm}], \quad \mathrm{L}=2[\mathrm{~m}]\end{array}$ & $\mathrm{AL}_{2} \mathrm{O}_{3} /$ Water & $\begin{array}{c}\text { CHF } \\
\text { enhancement } 32 \%\end{array}$ \\
\hline $\begin{array}{l}\text { A.A. R. Darzi et al. } \\
\text { [24] }\end{array}$ & $\begin{array}{l}\text { Steel tube }, \mathrm{Di}=8.1[\mathrm{~mm}] \\
\mathrm{L}=0.220[\mathrm{~m}]\end{array}$ & $\mathrm{S}_{\mathrm{i}} \mathrm{O}_{2} /$ water & $\begin{array}{c}\mathrm{CHF} \\
\text { Enhancement }\end{array}$ \\
\hline R. B. Mansour et al. [7] & $\begin{array}{cl}\text { Copper tube, } & \mathrm{Di}=6.35[\mathrm{~mm}] \\
\mathrm{L}=2.24 \mathrm{~m}\end{array}$ & $\mathrm{AL}_{2} \mathrm{O}_{3} /$ Water & $\begin{array}{c}\text { CHF } \\
\text { Decreases }\end{array}$ \\
\hline $\begin{array}{l}\text { K. Henderson et al. } \\
\text { [12] }\end{array}$ & $\begin{aligned} \text { Copper tube, } \begin{aligned} \mathrm{Di} & =7.9[\mathrm{~mm}] \\
\mathrm{L} & =2[\mathrm{~m}]\end{aligned}\end{aligned}$ & $\mathrm{S}_{\mathrm{i}} \mathrm{O}_{2} / \mathrm{R}-134 \mathrm{a}$ & $\begin{array}{l}\text { Heat transfer Coefficient Decreases } \\
55 \%\end{array}$ \\
\hline $\begin{array}{l}\text { I.M. Mahbubul et al. } \\
\text { [13] }\end{array}$ & smooth circular tube & $\mathrm{AL}_{2} \mathrm{O}_{3}-\mathrm{R} 141 \mathrm{~b}$ & $\begin{array}{c}\text { Heat transfer } \\
\text { Coeff. Increases }\end{array}$ \\
\hline
\end{tabular}




\subsection{Bubble dynamics in flow boiling}

Boiling occur when the temperature of the heater surface exceeds the saturation temperature, thus causing bubble formation. Bubble form in small pits and/or cavities on heater surface filled with vapour. These vapour filled cavities are called nucleation sites. Bubble growth is affected by strong temperature and velocity gradients [29].In vertical annular test section with increase in liquid velocity result in decrease in bubble size and lifetime. R. Situ et al. [32] did experimentally for bubble departure frequency in forced convective sub cool boiling flow. Water was used as the testing fluid, and the tests were performed at atmospheric pressure. A highspeed digital video camera was applied to capture the dynamics of the bubble nucleation process. R. Ahmadi et al. [31] studied experimentally bubble dynamics at boiling incipience in subcooled upward flow boiling; two types of bubble behaviour were observed after nucleation lift-off from the heated surface followed by collapsing rapidly in subcooled bulk liquid due to condensation, and sliding along the vertical heated surface for a long distance. Since the bubble lift-off was observed only when the wall superheat was high, the boundary between the lift-off and the sliding could be determined in terms of the Jakob number. So from previous research bubble growth rate, bubble frequency, these factors are affected in heat transfer coefficient in flow boiling.

\subsection{Effect of dissolved gases on flow boiling heat transfer}

The solubility of dissolved gases in liquids decreases with an increase in liquid temperature. This causes release of dissolved gases as the liquid temperature is raised resulting in an early nucleation at temperatures well below saturation temperature. The effect of dissolved gases on boiling heat transfer of water revived by S. G. Kandlikar [23], results indicate that the dissolved gases have no effects on the single-phase heat transfer. In the partial boiling region, the heat transfer coefficient increases due to increased nucleation activity.

\section{Conclusion}

1. The representative results and the review of the findings from the literature on convective heat transfer of nanofluids demonstrated that nanofluids exhibit an enhanced heat transfer coefficient compared to its base fluid and it increases significantly with increasing concentration of nanoparticles as well as Reynolds number.

2. From the review of available experimental results for boiling heat transfer, it can be conferred there is undisputed substantial increase in the boiling critical heat flux of nanofluids. However, reported data are still limited to clearly understand the underlying mechanisms and trend of boiling heat transfer performance of nanofluids.

3. This paper also presents various nanofluids formulation methods its advantages and limitations.

4. There are only a few results for CHF enhancement observed during flow boiling with nanofluids.

5. We need nanofluids that maintain their stability over increased operating times.

6. Much research has done with $\mathrm{AL}_{2} \mathrm{O}_{3}$ and very few research done with other type of nanofluids.

\section{References}

[1] H.S.Ahn, M. H. Kim, A Review on Critical Heat FluxEnhancement with Nanofluids andSurface Modification, Journal of Heat transfer, February 2012 Vol.134

[2] D.Wen, G.Lin, S.Vafaei, K.Zhang, Review of nanofluids for heat transfer applications, Particuology 7 (2009) p.141-150

[3] S.M.S.Murshed, C.A.N. D.Castro, M.J.V.Lourenco, M.L.M.Lopes, F.J.V.SantosA review of boiling and convective heat transfer with nanofluids, Renewable and Sustainable Energy reviews 15(2011) p.2342-2354

[4] J. Barber, D.Brutin, L. Tadrist, A review on boiling heat transfer enhancement with nanofluids, Nanoscale research letter a springer open letter 2011, 6:280

[5] T.Kim, Y. H. Jeong, S. H. Chang, An experimental study on CHF enhancement in flow boiling using $\mathrm{AL}_{2} \mathrm{O}_{3}$ nano-fluid, International Journal of Heat and Mass Transfer 53 (2010) p.1015-1022

[6] S. Suresh, K.P. Venkitaraj, P. Selvakumar, M. Chandrasekar,Effect of $\mathrm{AL}_{2} \mathrm{O}_{3}-\mathrm{Cu} /$ water hybrid nanofluid in heat transfer,Experimental Thermal and Fluid Science 38 (2012) p.54-60

[7] R. B. Mansour , N. Galanis , C.T. Nguyen,Experimental study of mixed convection with watereAl2O3 nanofluidin inclined tube with uniform wall heat flux, International Journal of Thermal Sciences 50 (2011) p.403-410

[8] S. Kakaç, A. Pramuanjaroenkij, Review of convective heat transfer enhancement with nanofluids, International Journal of Heat and Mass Transfer 52 (2009) p.3187-3196

[9] S. Z. Heris, M. N. Esfahany, S.G. Etemad, Experimental investigation of convective heat transferof Al2O3/water nanofluid in circular tube, International Journal of Heat and Fluid Flow 28 (2007) p.203-210

[10] M.M. Heyhat, F. Kowsary, A.M. Rashidi, M.H. Momenpour, A. Amrollahi,Experimental investigation of laminar convective heat transfer and pressure dropof water-based $\mathrm{Al}_{2} \mathrm{O}_{3}$ nanofluids in fully developed flow regime, Experimental Thermal and Fluid Science 44 (2013) p.483-489

[11] N. Singh, V. Sathyamurthy, W. Peterson, J. Arendt, D. Banerjee, Flow boiling enhancement on a horizontal heater using carbon nanotube coatings, International Journal of Heat and Fluid Flow 31 (2010) p. 201-207

[12] K. Henderson, Y.G. Park, L. Liu, A. M. Jacobi,Flow-boiling heat transfer of R-134a-based nanofluids in a horizontal tube, International Journal of Heat and Mass Transfer 53 (2010) p.944-951

[13] I.M. Mahbubul, R. Saidura,b, M.A. Amalinaa,Heat transfer and pressure drop characteristics of $\mathrm{Al}_{2} \mathrm{O}_{3}-\mathrm{R} 141 \mathrm{bnano}$ refrigerant in horizontal smooth circular tube,Procedia Engineering 56 ( 2013 ) p. 323 - 329

[14] X. Wang, A. S. Mujumdar, Heat transfer characteristics of nanofluids: a review, International Journal of Thermal Sciences 46 (2007) 1-19 
[15] H.Peng, G. Ding, W.Jiang, H. Hu, Y. Gao,Heat transfer characteristics of refrigerant-based nanofluidflow boiling inside a horizontal smooth tube, International journal of refrigeration 32(2009) p.1259-1270

[16] M.N.Golubovic, H.D.M. Hettiarachchi, W.M. Worek ,W.J. Minkowycz,Nanofluids and critical heat flux, experimental and analytical study ,Applied Thermal Engineering 29 (2009) p.1281-1288.

[17] V. Uskokovic,Nanotechnologies: What we do not know, Technology in Society 29 (2007) p.43-61.

[18] E. Abedini, A. Behzadmehr, S.M.H. Sarvari, S.H. Mansouri, Numerical investigation of subcooled flow boiling of a nanofluid,International Journal of Thermal Sciences 64 (2013) p.232-239.

[19] C.B. Tibiriçá, G. Ribatski, J. R.Thome,Saturated flow boiling heat transfer and critical heat flux in small horizontalflattened tubes,International Journal of Heat and Mass Transfer 55 (2012) p.7873-7883.

[20] S. J. Kima, T. McKrella, J. Buongiornoa, L. Hub,Subcooled flow boiling heat transfer of dilute alumina, zinc oxide, and diamondnanofluids at atmospheric pressure, Nuclear Engineering and Design 240 (2010) p.1186-1194.

[21] W. Yu, H. Xie,A Review on Nanofluids: Preparation, Stability Mechanisms, and Applications,Journal of NanomaterialsVolume 2012.

[22] S. G. Kandlikar,A General Correlation for SaturatedTwo-Phase Flow Boiling HeatTransfer Inside Horizontal and Vertical tubes, Journal of Heat Transfer, FEBRUARY 1990, Vol. 112

[23] S. G. Kandlikar, M. Bulut,An Experimental Investigation onFlow Boiling of Ethylene-Glycol/Water Mixtures, Journal of Heat Transfer, APRIL 2003, Vol. 125

[24] A.A. R. Darzi , M. Farhadi , K. Sedighi , R. Shafaghat , K. Zabihi,Experimental investigation of turbulent heat transfer and flow characteristics of $\mathrm{S}_{\mathrm{i}} \mathrm{O}_{2}$ /water nanofluid within helically corrugated tubes, International Communications in Heat and Mass Transfer 39 (2012)p.1425-1434

[25] E. P. B. Filho, L. Cheng, J. R. Thome,Flow boiling characteristics and flow pattern visualizationof refrigerant/lubricant oil mixtures, International journal of refrigeration 32 ( 2009) p.185-202

[26] S.Saisorn, J. KaewOn, S. Wongwises,Flow pattern and heat transfer characteristics of R-134a refrigerantduring flow boiling in a horizontal circular mini-channel, International Journal of Heat and Mass Transfer 53 (2010) p.4023-4038

[27] X.J. Wang, X.F. Li, N. Wang, X.Y. Wen, Q. Long, Influence of SDBS on stability of Al2O3 nano-Suspensions,Proc. of SPIE Vol. 6831

[28] R. Maurus, T. Sattelmayer, Bubble and boundary layer behaviour in subcooled flow boiling, International Journal of Thermal Sciences 45 (2006) p.257-268

[29] V.Proanovic, D. Fraser, M. Salcudean,Bubble behaviour in subcooled boiling flow boiling of water at low pressures and low flow rates, International journal of multiphase flow 28(2002) p.1-19

[30] R. Situ , M. Ishii , T. Hibiki ,J.Y. Tu , G.H. Yeoh , M. Mori, Bubble departure frequency in forced convective subcooled boiling flow, International Journal of Heat and Mass Transfer 51 (2008) p.6268-6282

[31] R. Ahmadi, T. Ueno, T. Okawa, Bubble dynamics at boiling incipience in subcooled upward flow boiling,International Journal of Heat and Mass Transfer 55 (2012) p.488-497

[32] R. Situ, T. Hibiki, M. Ishii, M. Mori, Bubble lift-off size in forced convective subcooledboiling flow, International Journal of Heat and Mass Transfer 48 (2005) p.5536-5548 